Www.jmscr.igmpublication.org

Index Copernicus Value: 79.54

ISSN (e)-2347-176x ISSN (p) 2455-0450

crossrefDOI: https://dx.doi.org/10.18535/jmscr/v7i2.16

\title{
Correlation of Clinicopathological Features of Breast Cancer with Its Intrinsic Subtypes
}

\author{
Authors \\ Dipanshu Kakkar ${ }^{1}$, R.K Karwasra ${ }^{2}$, Shubra Kochar ${ }^{3}$ \\ ${ }^{1}$ Junior Resident, Department of General and Oncosurgery, Pt. B.D.Sharma, PGIMS, Rohtak \\ ${ }^{2}$ Senior Professor and Head of Department of General and Oncosurgery, Pt.B.D.Sharma, PGIMS Rohtak \\ ${ }^{3}$ Senior Resident, Department of General Surgery, Pt. B.D.Sharma, PGIMS, Rohtak
}

\begin{abstract}
Background: Estrogen receptor (ER), Progesterone receptor $(P R)$ and Her2Neu expression status have important roles in prognosis and treatment of breast cancer. This study aims at correlating clinical and pathological features of breast cancer patients with its intrinsic subtypes.

Methods: 100 patients of breast cancer undergoing modified radical mastectomy were selected for the study. Four subtypes based on immunohistochemistry evaluated ER/PR/HER2 status, clinicopathological features, and prognosis were analyzed retrospectively and statistically.

Results: The following distribution was observed : luminal A subtype (ER+, PR+, Her2neu-) - 34 cases, luminal B subtype (ER+,PR+, Her2Neu+) -13 cases, Her2neu enriched (ER-, PR-, Her2Neu++)- 20 cases, basal subtype(triple negative)-33 cases. The clinical features like age, menarche age, parity, years of lactation and presence of risk factors were strongly correlated to breast cancer intrinsic subtypes ( $p$ $<0.05)$ while pathological factors like proliferative index is strongly correlated to breast cancer intrinsic subtypes $(p<0.05)$.

Conclusion: Breast cancer intrinsic subtypes are associated with its clinical and pathological features. This association can be helpful in treatment modification of patients with long term follow up. Study also shows the importance of NPI as a correct prognostic indicator and guide for planning adjuvant therapy that uses tumor size, nodal status and tumor grade.
\end{abstract}

\section{Introduction}

Cancer of the breast in women is a major health burden worldwide. It is the most common cause of cancer among women in both high-resource and low-resource settings, and is responsible for over one million of the estimated 10 million neoplasms diagnosed worldwide each year in both sexes. ${ }^{[1]}$ Breast cancer is the most common diagnosed malignancy in women worldwide $(22 \%)$ and in India $(18.5 \%)$ it ranks second to cervical cancer.
The burden of breast cancer is increasing in both developed and developing countries; the peak occurrence of breast cancer in developed countries is above the age of 50 whereas in India it is above the age of $40 .{ }^{[2]}$

\section{Clinicopathological Features}

Clinico-pathologic features of breast cancer includes - age at diagnosis, menopausal status, mean size of breast lump, presence of signs and symptoms, association with any risk factors, 
positive family history, history of chemotherapy or radiotherapy, history of hormonal therapy ,Tstage, presence of axillary lymph nodes, presence of distant metastasis, ER status, PR status, HER2 status, and histologic grade. ${ }^{[3]}$

Exposure to exogenous hormones as oral contraceptives $^{[4]}$ and hormone replacement therapy ${ }^{[5]}$ result in an increase in the risk of breast cancer. The risk conferred by oral contraceptive use is, however, rather small and although it persists for up to 10 years after cessation, cancers in these women, as with women taking hormone replacement therapy, are usually not clinically advanced at presentation, thus rendering the impact on mortality rather modest. ${ }^{[5]}$
Intrinsic Breast Tumor Subtypes and IHC Classification

Breast tumors may be classified using 5 immunohistochemical (IHC) tumor markers: estrogen receptor (ER), progesterone receptor (PR), human epidermal growth factor receptor 2 (HER2), HER1, cytokeratin 5/6 (CK 5/6) and ki67. Breast cancer is classified into four groups based on IHC profile ER/PR and Her2/neu expression, positive $(+)$ and/or negative $(-)$. The groups are:

- $\mathrm{ER} / \mathrm{PR}+$, Her2+ = ER+/PR+, Her2+; $\mathrm{ER}-/ \mathrm{PR}+, \mathrm{Her} 2+$; ER+/PR-, Her2+

- $\quad \mathrm{ER} / \mathrm{PR}+, \mathrm{Her} 2-=\mathrm{ER}+/ \mathrm{PR}+, \mathrm{Her} 2-$; ER-/PR+, Her2-; ER+/PR-, Her2-

- $\quad \mathrm{ER} / \mathrm{PR}-$, Her2+ = ER-/PR-, Her2+

- $\quad \mathrm{ER} / \mathrm{PR}-$, Her2- = ER-/PR-, Her2-

\section{Association of clinicopathologic features of breast cancer with intrinsicsubtype ${ }^{[6]}$}

\begin{tabular}{|c|c|c|c|c|}
\hline Intrinsic Subtype & Luminal A & Luminal B & HER2 Enriched & Basal-like \\
\hline $\begin{array}{l}\text { Estrogen/progesterone } \\
\text { receptor expression }\end{array}$ & Positive - strong & Positive - variable & Positive or negative & Negative \\
\hline HER2 amplification & Rare & $\begin{array}{l}\text { Rare though small } \\
\text { percentage positive }\end{array}$ & Common & Negative \\
\hline Grade & Low-intermediate & Intermediate-high & Intermediate-high & High \\
\hline P53 mutation & Rare & Uncommon & Common & Common \\
\hline Ki67\% & Low & Intermediate-high & High & High \\
\hline DNA copy number & Diploid & Aneuploid & $\begin{array}{l}\text { Aneuploid; high genomic } \\
\text { instability }\end{array}$ & $\begin{array}{l}\text { Aneuploid; high genomic } \\
\text { instability }\end{array}$ \\
\hline $\begin{array}{l}\text { mRNA expression } \\
\text { signature }\end{array}$ & $\begin{array}{l}\text { High ER cluster; } \\
\text { low proliferation }\end{array}$ & $\begin{array}{l}\text { Lower ER cluster, } \\
\text { high proliferation }\end{array}$ & $\begin{array}{l}\text { High HER2 amplicon, high } \\
\text { proliferation }\end{array}$ & $\begin{array}{l}\text { Basal signature, high } \\
\text { proliferation }\end{array}$ \\
\hline
\end{tabular}

The IHC classification correlates well with intrinsic gene expression microarray categorization: ER/PR+, Her2+ with Luminal B; ER/PR+, Her2- with Luminal A; ER/PR-, Her2+with HER2 Enriched and ER/PR-, Her2with triple negative/basal-like tumors. ${ }^{[6]}$ Apart from lending itself to subtype analyses of tumor when fresh tissue is not available, the IHC classification has prognostic and therapeutic implications, is inexpensive and readily available $^{[6]}$.

\section{Expression and clinical features of basic intrinsic subtypes}

Luminal tumors- The luminal-like tumors express hormone receptors, with expression profiles reminiscent of the luminal epithelial component of the breast. ${ }^{[7]}$ These patterns include the expression of luminal cytokeratins 8/18, ER and genes associated with ER activation such as LIVI and CCND1. ${ }^{[7]}$ At least two subtypes exist within luminal-like tumors, i.e., luminal $\mathrm{A}$ and luminal B.

Luminal tumors are the most common subtypes among breast cancer, with luminal A being the majority. Luminal tumors response well to hormone therapy but poorly to conventional chemotherapy ${ }^{[6]}$. Treatment response differs between luminal subtypes.

Luminal A tumors could be adequately treated with endocrine therapy, while luminal B tumors which are more proliferative may benefit more from the combined therapeutic strategy of chemotherapy and hormonal treatment ${ }^{[6]}$. Other 
targeted approaches such as anti-angiogenic strategies were suggested to be effective for luminal tumors as well ${ }^{[6]}$.

Basal tumors - Basal tumors account for $60 \%$ to $90 \%$ triple negative cases ${ }^{[8]}$. Risk factors for this subtype include earlier menarche, high waist-tohip ratio, and a lack of breast-feeding together with high parity ${ }^{[10]}$. These tumors are associated with a lower disease-specific survival and a higher risk of local and regional relapse ${ }^{[11]}$. The metastasis pattern also separates basal tumors from the other breast cancers, with a tendency towards visceral organs (excluding bone) and less likely to involve lymph nodes ${ }^{[20]}$. Given the triple negative receptor status, basal tumors are not amenable to conventional targeted breast cancer therapies, leaving chemotherapy the only option in the therapeutic armamentarium ${ }^{[9]}$.

\section{HER2 over-expression tumors}

The intrinsic HER2 over-expression tumors refer to those identified using gene expression array, which is similar to the ER-PR-HER2+ (ER negative, PR negative, HER2 positive) subgroup by immunostaining or fluorescence in situ hybridization $^{[12]}$ (FISH). Though HER2 overexpression breast tumors carry a poor prognosis, they are sensitive to anthracycline and taxanebased neoadjuvant chemotherapy, with significantly higher pathological complete response than luminal breast tumors ${ }^{[9]}$. The poor prognosis of this subtype as well as the basal tumors seem to derive from a higher risk of early relapse among those without complete eradication of tumor cells, and cancers of these two classes are suggested to derive the most benefit from improvements in chemotherapy ${ }^{[11]}$. Unlike the basal tumors, molecularly targeted agents such as the anti-HER2 monoclonal antibody, trastuzumab, are available for HER2 over-expression cancers $[13,14]$

Thus this study aims at correlating clinical pathological and intrinsic subtypes in breast cancer patients and their effect on prognosis of premenopausal and post menopausal patients.

\section{Materials and Methods}

Clinical data of 100 patients of breast cancer undergoing operations (modified radical mastectomy) in the department of General Surgery, Pt. B.D. Sharma Post Graduate Institute of Medical Sciences, Rohtak was collected and study was performed in a prospective randomised manner. Patients who have previously undergone thoracic or axillary surgery or Patients who have been administered neo adjuvant chemotherapy without prior evidence of IHC marking on core needle biopsy were excluded from the study. After recording demographic data, history and physical examination was done and malignancy was confirmed either by fine needle aspiration cytology (FNAC) or core needle biopsy (CNB). Routine investigations and metastatic work up was performed and clinical TNM stage was assessed in all the patients. Patients undergoing upfront surgery and palliative mastectomy included in study. MRM done and in every patient apical nodes also cleared. Main specimen and apical nodes sent separately in pathology department for detailed gross and microscopic examination to ascertain its size, status of margin, histology of the tumor, lymphnode metastasis, modified Richards Bloomson's (MRB) score and Nottingham Prognostic Index (NPI). MRB grade was obtained by adding up the scores for tubule formation, nuclear pleomorphism and mitotic count. Each of which was given 1, 2 or 3 points. This resulted in a total score of between 3 and 9.The final grading was given as below.

1.3 to 5 points - grade I

2. 6 to 7 points-grade II

3.8 to 9 points-grade III.

The Nottingham Prognostic Index was calculated by using three prognostic factors - lymph node stage, tumor size and histologic grade. Index formula which was used is as follows-

NPI $=[$ Size $(\mathrm{cm}) \times 0.2]+[$ Lymph node stage $(1-$ $3)]+[\operatorname{grade}(1-3)]$.

According to NPI three prognostic groups were identified ; a good group with scores of less than 
3.4 ; a moderate group with scores of $3.4-5.4$; a poor group with score of over 5.4.

The histological type, tumor grade, mitotic count, lymph node involvement, estrogen and progesterone receptor and Her-2/neu status were assessed. The tumor size was recorded from the histopathological reports. The ER and PR results were screened manually and interpreted as positive when more than $10 \%$ of tumor cells showed positive nuclear staining, similar to previous studies. $(15,16) \mathrm{Her}-2 / \mathrm{Neu}$ expression scored over DAKO score and a score of $3+$ and more considered positive.

\section{Statistical Analysis}

At the end of the study, the data will be collected and analysed statistically by using Student t-test and Chi-square test. The differences of clinicopathological characteristics in histological type, lymph node metastasis and the expression of biomarkers between the $\mathrm{BC}$ subtypes were evaluated by chi-square test. A p value of $<0.05$ will be considered as significant at a $95 \%$ confidence interval.

\section{Results}

Age of patients included in study ranged from 27 to 75 . Mean age of study was $52.22 \pm 10.062$ Years. Out of 100 study patients 99 patients are female and 1 is male patient. Mean B.M.I of patients is $21.819+/-3.3559$ (ranges 16.9 to 28.3 $\mathrm{kg} / \mathrm{m}^{2}$ ).Majority of the patients were in stage II $(\mathrm{n}=46,46 \%)$ and stage III $(\mathrm{n}=41,41 \%)$ while only few patients were in stage $\mathrm{I}(\mathrm{n}=11,11 \%)$ and stage IV $(n=2,2 \%)$.Most no. of patients attained menarche at an early age ( $<13$ years) $(n=62$, $62 \%$ ).

Most no. of patients were post menopausal ( $\mathrm{n}=64$, $64 \%)$. A large no. of patients $(n=77,77 \%)$ attained pregnancy at an early age. Less than half no. of patients $(n=42,42 \%)$ had more than 3 off springs in their lifetime (parity $>3)(\mathrm{n}=55,55 \%)$ and an equal no. of patients had history of lactation $>6$ months. Almost half no. of patients $(\mathrm{n}=58,58 \%)$ had positive history of risk factors in their lifetime.

Tumor size was available for all 100 patients, in which $25(25 \%)$ samples were less than $2 \mathrm{~cm}, 58$ $(58 \%)$ were within $2-5 \mathrm{~cm}$, and $17(17 \%)$ were more than $5 \mathrm{~cm}$. The pathology assessment indicated that 70 cases $(70 \%)$ had involved in lymph node metastasis. Among the patients, 77 $(77 \%)$ were in tumor grade I, $15(15 \%)$ in grade II and $8(8 \%)$ cases in grade III. All patients were invasive ductal carcinoma. Majority of the patients(n=62, 62\%) had moderate NPI score ( Nottingham prognostic index). Among all patients, 42 patients $(42 \%)$ were ER positive, 46 (46\%) patients were PR positive and only 33 (33\%) patients were Her2Neu positive. Half of the patients $(n=50,50 \%)$ have high proliferative index (Ki67).

Among the luminal subtypes, maximum no. of patients $(n=34,34 \%)$ were of luminal A subtype, while least no. of patients $(n=13,13 \%)$ were of luminal B subtype.

Table 2 Intrinsic subtypes distribution in breast cancer patients

\begin{tabular}{|c|c|c|c|c|c|}
\hline \multicolumn{2}{|c|}{} & Frequency & Percent & Valid Percent & Cumulative Percent \\
\hline \multirow{4}{*}{ Valid } & BASAL & 33 & 33.0 & 33.0 & 33.0 \\
\cline { 2 - 6 } & HER2 E & 20 & 20.0 & 20.0 & 53.0 \\
\cline { 2 - 6 } & A & 34 & 34.0 & 34.0 & 87.0 \\
\cline { 2 - 6 } & B & 13 & 13.0 & 13.0 & 100.0 \\
\cline { 2 - 6 } & Total & 100 & 100.0 & 100.0 & \\
\hline
\end{tabular}

\section{Comparison of clinical features of breast cancer with intrinsic subtypes}

A linear correlation is made between clinical features of breast cancer with its intrinsic subtypes. Intrinsic subtypes were directly proportional to age of the patients ( $p$ value $<0.05$ ) and B.M.I of the patients ( $\mathrm{p}$ value $=0.0001$ ). Basal subtype $(55 \%)$ was found to be higher in patients with $<45$ years of age while luminal A subtype (36.3\%) was found to be higher in patients with $>45$ years of age. According to our observations, it was found that menarche age ( $p$ value $=0.002)$, 
age of first pregnancy ( $\mathrm{p}$ value $=0.004)$, parity and years of lactation ( $\mathrm{p}$ value $=0.001$ ) were strongly correlated to breast cancer intrinsic subtypes. Correlation between risk factors ( $\mathrm{p}$ value $=0.0001$ ) and intrinsic subtypes was also found to be strong with luminal A subtype (71.4\%) having most strong correlation with the presence of risk factors. Correlation between intrinsic subtypes and clinical features like nipple discharge, family history and menopausal status was not found to be significant ( $\mathrm{p}$ value $>0.05$ )

Comparison of pathological features of breast cancer with intrinsic subtypes

Pathological features like lump size, presence of axillary lymph node metastasis, pathological stage, pathological grade, NPI score and proliferative index were included in the study. Correlation between pathological features like lump size, presence of axillary lymph node metastasis, pathological stage, pathological grade and NPI score with breast cancer intrinsic subtypes was not found to be significant(p value $>0.05)$. The proportion of any tumor size was found to be higher in basal subtype (35\%) and luminal A subtype (34.3\%) while lower in luminal B subtype $(16 \%)$ and her2Neu enriched subtype (17\%). Stage I group, most patients (36.4\%) belong to Basal subtype and Luminal $\mathrm{A}$ and Luminal B subtype (27.3\%). Stage II group, most patients belong to Luminal A subtype (37\%), and Basal subtype $(32.6 \%)$. In stage III group, most patients belong to Basal subtype (34.3\%) and Luminal A subtype (31.3\%). In stage IV patients, majority of the patients belong to Her2Neu enriched subtype (50\%). In Grade I patients, most patients belong to Luminal A subtype (37.7\%), and Basal subtype(31.2\%). Grade II patients, fall in the category of luminal A subtype (33.7\%) and her2neu enriched subtype (26.7\%). In Grade III patients, $6(75 \%)$ patients belong to Basal subtype while only $2(25 \%)$ patients belong to Her2 Neu enriched subtype. No patient in Grade III belong to Basal or Luminal B subtype.

In patients with excellent NPI score, 3 (42.9\%) each patients belong to Luminal $\mathrm{A}$ and Basal subtype, 1 (14.3\%) patients belong to Her2 Neu enriched subtype while no patient belong to Luminal B subtype. In patients with good NPI score, $9(42.9 \%)$ patients belong to luminal A subtype, $5(23.8 \%)$ patients belong to Luminal B subtype, 4(19\%) patients belong to Basal subtype while only 3 (14.3\%) patients belong to Her2Neu enriched subtype. In patients with moderate NPI score, $21(33.9 \%)$ patients belong to Luminal A and Basal subtype each, 14(22.6\%) patients belong to Her2 Neu enriched subtype, while only $6(9.7 \%)$ patients belong to Luminal B subtype. In patients with poor NPI score, 5(50\%) patients belong to Basal subtype, 2 (20) patients belong to Her2 Neu and Luminal subtype each, while only 1 (10\%) patient belong to Luminal A subtype.

In patients with high proliferative index group, 30 (60\%) patients are of Basal subtype, 17 (34\%) patients are of Her2 Neu enriched subtype, $3(6 \%)$ patients are of Luminal A subtype while no patient belong Luminal B subtype in this group. In patients with intermediate proliferative index, 10 (83.3\%) patients belong to Luminal B subtype, 2 (16.7\%) patients belong to Luminal A subtype while no patient belong to Basal or Her2Neu enriched subtype in this group. In patients with low proliferative index, 29 (76.3\%) patients belong to Luminal A subtype, while 3 (7.9\%) patients each belong to Her2 Neu enriched, Luminal B subtype and Basal subtype. According to our observations, Luminal B and Luminal A patients have low or intermediate proliferative index while Basal and Her2 Neu enriched tumors have high proliferative index.

\section{Discussion}

In the present study, The majority of patients in the study belong to 46-60 years of age group, this shows the incidence of carcinoma breast in this age group in Indian population which is a decade earlier than the western countries. The mean age is lower in the study conducted in our department previously, Sandhu et al 2010. ${ }^{[17]}$ Western patients have a higher age at presentation Stead et al 2009. [16] 
The incidence of luminal A subtype is maximum in this age group (36.8\%). Incidence of Basal and Her2 neu Enriched subtype decreases with increasing age although this relationship is not linear. This correlation was found to be significant $(\mathrm{p}<0.001)$

Majority patients in our study are found to be in normal range of B.M.I (45\%). Luminal B and Her2-type cases are found to be underweight (BMI $<18.5 \mathrm{~kg} / \mathrm{m}^{2}$ ) while luminal A and Basal subtype patients are either normal weight (BMI 18.5-24.99) or overweight (BMI>25). This correlation was found to be highly significant. $(\mathrm{p}<0.0001)$.

The observations are found to be according to literature which says that luminal B and Her2-type cases are likely to be underweight (BMI $<18.5$ $\mathrm{kg} / \mathrm{m}^{2}$ ) while luminal A and basal subtype patients are either normal weight(BMI= 18.5-24.99) or overweight $\left(\right.$ BMI > 25) $\left(\right.$ Kosei Kimura et al) ${ }^{[18]}$

Most patients (77\%) in our study had their first pregnancy at or before age of 22 years i.e pregnancy at early age. Out of the patients who have their first pregnancy at early age, majority patients (39\%) are of Basal subtype. In patients who have their first pregnancy at late age, majority patients (42.9\%) are of Luminal A subtype. This correlation is found to be Highly Significant $(\mathrm{p}<0.001)$. According to literature, [Fataneh Zeiyaie et al] ${ }^{[19]}$ increased age at first birth is strongly associated with hormone receptor + tumors (Luminal A and Luminal B) while decreased age at first birth is associated with triple negative breast cancers ( Basal subtype).

Majority patients in our study attained Menarche at or before age of 13 years (62\%) which is considered as Early Menarche. In patients who attained Early Menarche maximum patients (39.7\%) are of Luminal A subtype and patients who attained Menarche after 13 years of age, maximum no. of patients $(56.8 \%)$ are of Basal subtype. This correlation is found to be Highly Significant $(\mathrm{p}<0.001)$. Fataneh Zeiyaie et $\mathrm{al}^{[19]}$ recorded the same results.
Most patients in our study are Postmenopausal (64\%). Out of post menopausal patients majority patients are of Basal and Luminal A subtype (31.3\% each ).In Pre menopausal patients majority are of similar Luminal subtypes again i.e Basal and Luminal A. The correlation was found to be Not Significant $(p>0.05)$. Lisa A Carey et $\mathrm{al}^{[20]}$ concluded that Basal-like breast tumors occurred at a higher prevalence among premenopausal African American patients compared with postmenopausal African American patients and Luminal A subtype occur at higher prevelance in postmenopausal non African American women compared to premenopausal non African American women while Her2 Neu enriched subtype has no significant correlation with menopausal status of patients

Majority patients in our study had $<3$ offsprings in their lifetime $(55 \%)$. Women who have parity $>3$ in our study, largely of them are of Basal subtype (60\%) followed by Her2 Neu Enriched (36.4\%). On the other hand, women who have parity $<3$, majority of them are of Luminal A subtype (70.5\%). The correlation was found to be Highly Significant $(\mathrm{p}<0.001)$.Xuezheng Sun et al ${ }^{[21]}$ states that high parity (3+births) and recent birth are strongly associated with luminal tumors (luminal A and luminal B subtypes)

$55 \%$ of patients in our study have breastfed their children $<6$ months in their lifetime. Majority of women in the same group are of Basal subtype (60\%) followed by Her2 Neu Enriched subtype (36.4\%). The correlation was found to be Highly Significant $(\mathrm{p}<0.001)$.

Risk factors like consumption of alcohol, previous radiation exposure, hormone replacement therapy are considered in this study. $58 \%$ of patients had no risk factors in their life time, out of which majority have basal intrinsic subtype (50\%) followed by Her 2 Neu Enriched (27.6\%). On the other hand those who have previous exposure of risk factors have Luminal A as their main subtype (71.4\%). The correlation was found to be Highly Significant $(\mathrm{p}<0.0001)$. Xiaohong R. Yang et al ${ }^{[22]}$ study concluded that Luminal A tumors were 
associated with most exposures that have been consistently related to overall breast cancer risk. $70 \%$ patients had axillary metastasis. Out of which majority $(34.3 \%)$ are found to be Basal subtype (34.3\%) followed by Luminal A (32.9\%). Patients with no axillary lymph node metastasis have Luminal $\mathrm{A}$ as their major intrinsic subtype (36.7\%) followed by Basal subtype (30\%). This correlation was found to be Not Significant $(p>0.05)$. Study by Godwin A Ebugheet $\mathrm{al}^{[23]}$ concluded that Her2Neu overexpression (her2neu enriched) is associated with higher incidence of lymph node metastasis.

In the present study, majority patients presented with stage II (46\%) followed by stage III $(41 \%)$ followed by stage I (11\%) and lastly stage IV (2\%). With increasing stage, incidence of Luminal B subtype is decreasing, while incidence of Her $2 \mathrm{Neu}$ subtype is increasing. The incidence of Luminal A and Basal subtypes is almost similar in stage I,II and III. The correlation was found to be Not Significant(p>0.05). I Mehdi et $\mathrm{al}^{[24]}$ concluded that women aged $>65$ years at the time of diagnosis reported more early stage tumors in the Luminal subtypes, and more stage III tumors in the ERBB2, basal, and unclassified subtypes.

Maximum patients belonged to Grade I (77\%) followed by Grade II (15\%) and grade III (8\%). Majority of patients in Grade 1 belongs to Luminal A subtype (37.7\%) followed by Basal subtype (31.2\%). With increasing Grade, incidence of Basal subtype increases and Luminal A subtype decreases, although this relationship isn't linear. This correlation was found to be Not Significant Statistically $(p>0.05)$. Lisa A Carey et $\mathrm{al}^{[20]}$ concluded that compared with Luminal A subtype, Basal subtype presents with higher histological grade (Grade 1 and 2). Siadati S et al [25] observed significant correlation of ER/PR expression (Luminal A and Luminal B) with grade 1 and 2 tumors while Her2 Neu overexpression (Her2 Neu subtype) is associated with grade 3 tumors.

In our study, majority patients have moderate NPI score $(62 \%)$ i.e Intermediate prognosis. Most of the patients with moderate NPI score are of Basal and Luminal A subtype (33.9\% each). The incidence of Luminal A subtype (42.9\%) and Luminal B (23.8\%) was found to be maximum in patients with good NPI score. This correlation is found to be Not Significant Statistically ( $p>0.05$ ). Hong chao zhen et $\mathrm{al}^{[26]}$ observed that ER positive expression and PR high expression were positively correlated with NPI. The percentages of cases with NPI score 2.00-3.40 were higher in the luminal A, ER+, PR high expression and Ki67 low expression group, and the percentages of cases with NPI > 5.40 were higher in the HER2 overexpression subtype, basal-like subtype, ER-, PR low/negative expression, and Ki67 high expression groups

ER and PR positivity was $42 \%$ and $46 \%$ respectively. $33 \%$ patients were Her-2/Neu positive.

In the present study, $50 \%$ patients are of high proliferative index, $12 \%$ patients are of intermediate proliferative index and $38 \%$ patients are of low proliferative index. The incidence of Basal subtype (60\%) and Her 2Neu subtype( 34\%) are found to be maximum in high proliferative index patients while incidence of Luminal B subtype $(83.3 \%)$ are found to be maximum in intermediate proliferative index patients. Incidence of Luminal A subtype (76.3\%) is found to be maximum in low proliferative index patients. This concludes that hormone receptor positivity increases with decreasing proliferative index. This correlation is found to be Highly Significant $(\mathrm{p}<0.001)$. Ivkovic Kapicl $\mathrm{T}$ et $\mathrm{al}^{[27]}$ concluded that Her2 Neu overexpression is associated with high proliferative index ( Ki67).

\section{Conclusion}

With increasing age of presentation of carcinoma breast, the incidence of Basal and Her $2 \mathrm{Neu}$ Enriched Luminal subtypes decreases which shows that elderly women usually have Luminal subtypes which have comparatively better prognosis i.e Luminal A and Luminal B. 
Clinical factors like obesity ( calculated in terms of BMI), age of Menarche, age of first Pregnancy, Parity, duration of lactation, presence of risk factors( intake of hormone replacement therapy, alcohol consumption, radiation exposure etc.) are strongly correlated with breast cancer intrinsic subtypes.

With increasing stage, incidence of Luminal B subtype is decreasing, while incidence of Her 2 Neu subtype is increasing. The incidence of Luminal A and Basal subtypes is almost similar in stage I,II and III though the correlation is not Statistically Significant.

With increasing grade, incidence of Basal subtype increases and Luminal A subtype decreases in our study.

Proliferative index is strongly correlated with breast cancer intrinsic subtypes. Hormone receptor positivity increases with decreasing proliferative index, this shows that High levels of Ki-67 are associated with worse prognoses.

Study also shows the importance of NPI as a correct prognostic indicator and guide for planning adjuvant therapy that uses tumor size, nodal status and tumor grade.

\section{References}

1. Ferlay J, Bray F, Pisani P, Parkin DM. GLOBOCAN 2000: Cancer Incidence, mortality and prevalence worldwide. IARC Cancer Base No. 5. 2001, Lyon, France: IARC.

2. Yeole BB, Kurkure AP. An epidemiological assessment of increasing incidence and trends in breast cancer in Mumbai and other sites in India, during the last two decades. Asian Pac J Cancer Prev 2003;4: 51-6.

3. Dr Narayan Das, Dr AnarshDebbarma, Dr Anup kumarSaha.Evaluation of ClinicoPathological Study of Breast Cancer in Rural PopulationIOSR Journal of Dental and Medical Sciences (IOSR-JDMS) eISSN: 2279-0853, p-ISSN: 2279-
0861.Volume 15, Issue 9 Ver. XI (September). 2016), PP 67-69

4. Grabrick DM, Hartmann LC, Cerhan JR, et al. Risk of breast cancer with oral contraceptive use in women with a family history of breast cancer. JAMA2000; 284:1791-1798

5. Pike MC, Krailo MD, Henderson BE, Casagrande JT, Hoel DG. 'Hormonal' risk factors, 'breast tissue age' and the ageincidence of breast cancer. Nature 1983;303:767-70.

6. Adapted from Cancer Genome Atlas network. Comprehensive molecular portraits of human breast tumours. Nature 2012;490:61-70

7. Smid M, Wang Y, Zhang Y, Sieuwerts AM, Yu J, Klijn JG, et al. Subtypes of breast cancer show preferential site of relapse. Cancer Res 2008;68:3108-3114.

8. Fan C, Oh DS, Wessels L, et al. Concordance among gene-expression-based predictors for breast cancer. N Engl J Med. 355(6):560-9, 2006.

9. Kohler BA, Sherman RL, Howlader N, et al. Annual report to the nation on the status of cancer, 1975-2011, featuring incidence of breast cancer subtypes by race/ethnicity, poverty, and state. J Natl Cancer Inst. 107(6), 2015.

10. Sineshaw HM, Gaudet M, Ward EM, et al. Association of race/ethnicity, socioeconomic status, and breast cancer subtypes in the National Cancer Data Base (2010-2011). Breast Cancer Res Treat. 145(3):753-63, 2014.

11. Voduc KD, Cheang MC, Tyldesley S, Gelmon K, Nielsen TO, Kennecke H. Breast cancer subtypes and the risk of local and regional relapse. $\mathrm{J}$ Clin Oncol. 28(10):1684-91, 2010.

12. Howlader N, Altekruse SF, Li CI, et al. US incidence of breast cancer subtypes defined by joint hormone receptor and HER2 status. J Natl Cancer Inst. 106(5), 2014. 
13. Schott AF. Systemic treatment for HER2positive metastatic breast cancer. https://www.uptodate.com/contents/systemi c-treatment-for-her2-positive-metastaticbreast-cancer? Last updated Jun 07, 2017. Accessed July 1, 2017.

14. Morrow M, Burstein HJ, Harris JR. Chapter 79: Malignant Tumors of the Breast. In: DeVita VT, Lawrence TS, Rosenberg SA, eds. DeVita, Hellman, and Rosenberg's Cancer: Principles and Practice of Oncology. 10th ed. Philadelphia, Pa: Lippincott Williams \& Wilkins; 2015.

15. Stead LA, Lash TL, Sobieraj JE, Chi DD, Westrup JL, Charlot M, et al. Triplenegative breast cancers are increased in black women regardless of age or body mass index. Breast Cancer Res. 2009;11(2):1-10.

16. Albergaria A, Ricardo S, Milanezi F, Carneiro V, Amendoeira I, Vieira D, et al. Nottingham Prognostic Index in TripleNegative Breast Cancer: A reliable prognostic tool? BMC Cancer [Internet]. 2011;11(1):299. Available from:http://www.biomedcentral.com/14712407/11/291

17. Sandhu D, Sandhu S, Karwasra R, Marwah S. Profile of breast cancer patients at a tertiary care hospital in north India. Indian J Cancer [Internet]. 2010;47(1):16. Available from:

http://www.indianjcancer.com/text.asp?201 0/47/1/16/58853.

18. Kosei Kimura, Satoru Tanaka,Mitsuhiko Iwamoto, Hiroya Fujioka, Yuko Takahashi , NayukoSatou, and Kazuhisha Uchiyama ;Association between body mass index and breast cancer intrinsic subtypes in Japanese women. Article in Experimental and therapeutic medicine 4(3):391-396 - September 2012

19. FatanehZeiyaie et al; The relationship of reproductive risk factors and histologic patterns with molecular subtypes of breast cancer International Journal of cancer management: June 2018,11(6); e66746published online:June 9,2018

20. Lisa A. Carey, MD; Charles M. Perou, $\mathrm{PhD}$; Chad A. Livasy, MD; et al Race, Breast Cancer Subtypes, and Survival in the Carolina Breast Cancer Study. JAMA. 2006;295(21):2492-2502

21. Xuezheng Sun, Hazel B. Nichols, Chiu-Kit Tse, Mary B. Bell, Whitney R. Robinson, Mark E. Sherman, Andrew F. Olshan, and Melissa A. Troester; Association of Parity and Time since Last Birth with Breast Cancer Prognosis by Intrinsic Subtype, 2015 American Association for Cancer Research

22. Xiaohong R.Yang, Mark E.Sherman, David L.Rimm, JolantaLissowska et al; Differences in Risk Factors for Breast Cancer Molecular Subtypes in a Population-Based Study. Cancer epidemiology biomarkers and prevention. March 2007Volume 16, Issue 3

23. Godwin A Ebughe et al, Histological; type and tumor grade in nigerian breast cancer: relationship to menarche, family history of breast cancer,parity,age at first birth, and age at menopause IOSRjournal of Dental and Medical Sciences(IOSR-JDMS) eISSN: 2279-0853p-ISSN:22790861.2013;7(5):58-63.

24. I Mehdi et al,breast cancer molecular subtypes in Oman:Correlation with age,histology, and stage distributionAnalysis of 542 cases national oncology center, the Royal Hospital, Muscat, Omangulf journal of oncology, The1(15):38-48.March 2014.

25. Siadati S, Sharbatdaran M, Nikbakhsh N, Ghaemian N. Correlation of ER, PR and HER-2/neu with other prognostic factors in infiltrating ductal carcinoma of breast. Iran J Pathol. 2015;10(3):221-6.

26. Hongchao Zhen, Liuting Yang, Li Li1, Junxian Yu, Lei Zhao, Yingying Li and Qin 
$\mathrm{Li}$; Correlation analysis between molecular subtypes and Nottingham Prognostic Index in breast cancer. Oncotarget, 2017, Vol. 8, (No. 43), pp: 74096-74105

27. Ivkovic-Kapicl T, Knezevic-Usaj S, DjilasIvanovic D, Panjkovic M. Correlation of HER-2/neu protein overexpression with other prognostic and predictive factors in invasive ductal breast cancer. In Vivo. 2007;21(4):673-8. 